This copy is for your personal, non-commercial use only.

If you wish to distribute this article to others, you can order high-quality copies for your colleagues, clients, or customers by clicking here.

Permission to republish or repurpose articles or portions of articles can be obtained by following the guidelines here.

The following resources related to this article are available online at www.sciencemag.org (this information is current as of May 30, 2013 ):

Updated information and services, including high-resolution figures, can be found in the online version of this article at:

http://www.sciencemag.org/content/340/6136/1090.full.html

Supporting Online Material can be found at:

http://www.sciencemag.org/content/suppl/2013/04/17/science.1234316.DC1.html

This article cites 17 articles, 3 of which can be accessed free:

http://www.sciencemag.org/content/340/6136/1090.full.html\#ref-list-1

This article appears in the following subject collections:

Ecology

http://www.sciencemag.org/cgi/collection/ecology 
M. J. Campos, D. Rother, G. Ambar, C. Dracxler, E. R. Castro, R. Laps, P. Develey, M. R. Francisco, and staff from PN Iguaçu for sending us palm seeds and data on frugivores; Fundação Florestal for allowing our study in the Protected Areas; and S. Nazareth and R. Brandolim for field and laboratory assistance. M.G. and P.R.G. receive a research fellowship from Conselho Nacional de Desenvolvimento Científico e Tecnológico.
Data supporting this study are available in the DRYAD repository (http://dx.doi.org/10.5061/dryad.2pm42). Bird plates were done by Carl Buell.

Supplementary Materials

www.sciencemag.org/cgi/content/full/340/6136/1086/DC1

Materials and Methods
Figs. $\mathrm{S} 1$ to $\mathrm{S} 4$

Tables $\mathrm{S} 1$ to $\mathrm{S} 6$

References (27-61)

7 December 2012; accepted 5 April 2013

10.1126/science.1233774

\section{Tracking Individuals Shows Spatial Fidelity Is a Key Regulator of Ant Social Organization}

\author{
Danielle P. Mersch, ${ }^{1 *}$ Alessandro Crespi, $^{2}$ Laurent Keller $^{1 *}$
}

Ants live in organized societies with a marked division of labor among workers, but little is known about how this division of labor is generated. We used a tracking system to continuously monitor individually tagged workers in six colonies of the ant Camponotus fellah over 41 days. Network analyses of more than 9 million interactions revealed three distinct groups that differ in behavioral repertoires. Each group represents a functional behavioral unit with workers moving from one group to the next as they age. The rate of interactions was much higher within groups than between groups. The precise information on spatial and temporal distribution of all individuals allowed us to calculate the expected rates of within- and between-group interactions. These values suggest that the network of interaction within colonies is primarily mediated by age-induced changes in the spatial location of workers.

A nt colonies, with their complex and efficient social organization, have long fascinated humans (1). Essential to their ecological success are high levels of cooperation and sophisticated division of labor. Although workers must perform a multitude of tasks such as foraging, nest construction, and brood rearing, it has become clear that there is no central control of how work is allocated among individuals. Therefore, workers must allocate themselves to tasks in a self-organized manner following simple behavioral rules that incorporate local stimuli received directly from the environment and from interactions with other workers (2-4). Despite extensive work on division of labor in social insects $(1,3,5-7)$, the connection between individual task specialization and the social network remains unknown. Another important, yet little studied aspect of social organization is spatial organization. In honeybees, workers change tasks over the course of their lifetime, starting as nurses in the nest and generally ending as foragers outside $(2,8,9)$. This suggests that the rate of interactions between group members may be affected by the task performed and its associated localization in the colony. In our experiment, we used an automated video tracking system based on fiducial identification labels to track all individuals in six colonies of the ant Camponotus fellah and to identify individual interactions and patterns of social organization (movie S1).

${ }^{1}$ Department of Ecology and Evolution, University of Lausanne, Switzerland. ²Biorobotics Laboratory, Ecole Polytechnique Fédérale de Lausanne, Switzerland.

*Corresponding author. E-mail: danielle.mersch@unil.ch (D.P.M.); laurent.keller@unil.ch (L.K.)
All colonies were established from a single queen collected after a mating flight. The experiment started when queens were 4 years old, out of a maximum life span of 26 years $(10)$. We determined the age of all workers (122 to 192 per colony) by weekly color-coding all newly eclosed workers more than 60 weeks before the experiment began. A month before the start of the experiment, we individually marked all ants with a distinct barcode-like matrix (11), enabling individual identification (12). Colonies were kept in a constantly dark nest chamber that was connected by a tunnel to a foraging chamber exposed to daily light-dark cycles (fig. S1). The temperature, humidity, light, and food supply were computer-controlled, and both chambers were filmed from above with high-resolution monochrome cameras operating under infrared light (fig. S1) (12). We recorded the position and orientation of all individuals twice per second to reconstruct spatial movement and infer all social interactions occurring over the 41 days of the experiment. A pair of ants was considered to interact when the front end of one ant was located within the trapezoidal shape representing the other ant (fig. S4) (12). The data set we obtained consisted of a total of 2,433,250,580 ant positions and 9,363,100 social interactions (movies S2 and S3).

We used this data set to first investigate whether workers organize themselves into cohesive social groups by using the Infomap community detection algorithm (13). To facilitate data analysis, we split the 41 experimental days into four periods of $11,10,10$, and 10 days. In each of these periods, we identified pairs of interacting ants. Analyses on the daily interaction networks of the first 11 days (see supplementary text) revealed two robust groups to which the same set of workers was affiliated on almost all days. The first group always comprised the queen and $41 \pm$ $12 \%$ of the workers (percentage \pm SD across the six colonies) (Fig. 1A), whereas the second group represented $31 \pm 11 \%$ of the colony's workforce. Depending on days and colonies, we also identified zero to five other groups of workers. A visual analysis of the daily networks of interactions suggested that workers affiliated with the two robust groups on only a few days may form a third group with less marked within-group preferential interactions. These workers represented $28 \pm 4 \%$ of the colony's workforce and were consistently located between the two other groups in the network (Fig. 1A and figs. S6 to S10). An additional analysis of the interaction frequencies (supplementary text) confirmed that workers of the third group interacted significantly more with members of their group than with workers of the other two groups. Together, these results indicate that colonies of C. fellah are structured in three interconnected social groups and that these groups differ in their interaction patterns.

Workers from the three social groups exhibited distinct behavioral signatures (Fig. 2). Workers of the first group performed most of the interactions with the queen [Kruskal-Wallis $\left.(\mathrm{KW}): \chi^{2}=514.05, P<10^{-101}\right]$ and visits to the brood. By contrast, workers from the second group performed most $(87.3 \pm 18.6 \%)$ of the foraging trips, whereas workers from the third group exhibited a significantly higher propensity to visit the rubbish pile. For simplicity, we hereafter refer to these three groups as nurses, foragers, and cleaners. Comparison of the normalized age of workers revealed an age-based division of labor. Nurses were younger than cleaners who, in turn, were younger than foragers $\left(\mathrm{KW}: \chi^{2}=108.7\right.$, $P<10^{-23}$ ) (fig. S11). However, in all colonies, there was great overlap among the three groups, with some nurses being older and some foragers being younger than the workers' average age. Despite a wide distribution in worker body size (6 to $16 \mathrm{~mm}$ ), no consistent size difference exists between workers of the three groups (fig. S12).

Our data also allowed us to track temporal changes among the three behavioral groups by performing community detection analyses on the three subsequent 10-day periods of the experimental data (Fig. 3). Workers exhibited a preferred behavioral trajectory, moving from nursing to cleaning to foraging as they age. The most common transition was from cleaner to forager (supplementary text). Such age-related behavioral transitions have been documented in honeybees, in which young bees nurse the brood, then move on to perform various other in-hive tasks and 
finally become foraging bees $(8,9)$. In ants, age polyethism is also believed to exist, but this is generally based on indirect evidence [reviewed in (1), but see (14) and supplementary text], with the notable exception of one study of Lasius niger, where individual marking of 40 workers revealed a progressive increase of the proportion of foragers over time, but where only $50 \%$ of the workers exhibited a behavioral age-related transition (15). Our data further emphasize that age-related behavioral maturation is a slow and noisy process in ants with important individual variation.

There were differences among the three groups in the frequency of within-group interactions. In particular, the relative within-group interaction frequency of cleaners was 2.1 times lower than that of nurses and foragers $\left(\mathrm{KW}: \chi^{2}=270.6, P<\right.$
$10^{-53}$ ), indicating that cleaners' group cohesion is less pronounced. This pattern was confirmed by an analysis considering the time required for a worker to interact with $80 \%$ of its nestmates (supplementary text). The observed social group structure and increased within-group interactions could stem from either a propensity of individuals to preferentially interact with members of their group or from differences in the spatial distribution of the different groups. We therefore used spatial grid representations (heat maps) to determine the spatial distribution of workers of each of the three groups over the first 11 days of observation. The heat maps revealed marked differences between nurses, cleaners, and foragers, which occupied distinct spatial locations in the nest area (Fig. 4A and figs. S13A to S17A).
Nurses stayed near the brood and foragers near the nest entrance, whereas cleaners occupied the remaining nest areas, which suggests that, in addition to cleaning, they might also patrol the nest.

Similar heat maps for the location of social interactions within and between each of the three groups revealed that most of the within-group interactions occurred in the spatial area most used by individuals of a given group, whereas betweengroup interactions preferentially occurred at the intersection of the spatial distribution of the pairs of groups considered (Fig. 4B and figs. S13B to S17B). To test whether the social structure and network of interaction might arise solely as a consequence of task-related spatial segregation of workers, we estimated for each worker the expected frequency of interactions within and

A
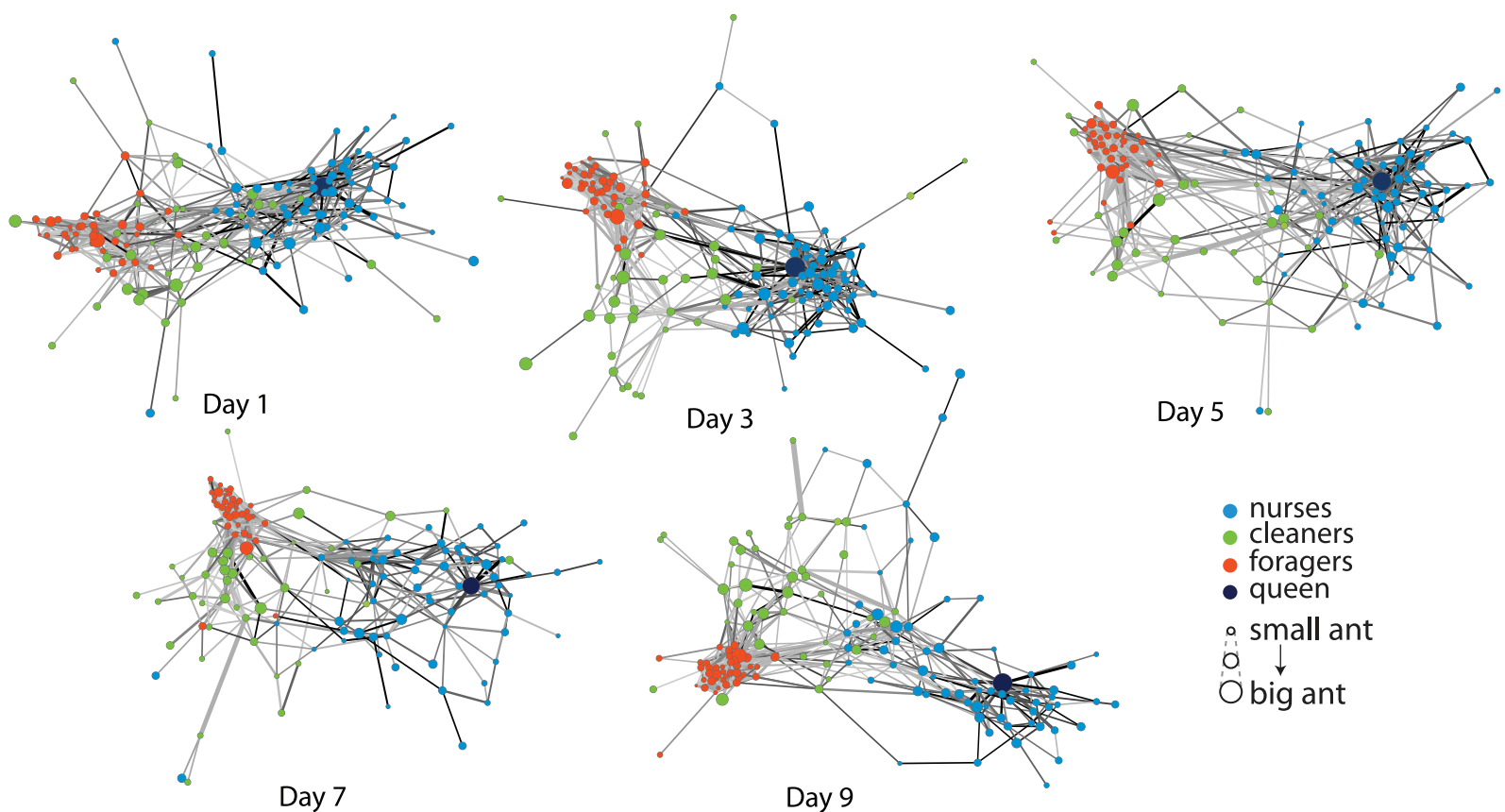

- nurses

- cleaners

- foragers

- queen

a small ant

o. $\downarrow$

Obig ant

B

Interaction frequency of nurses

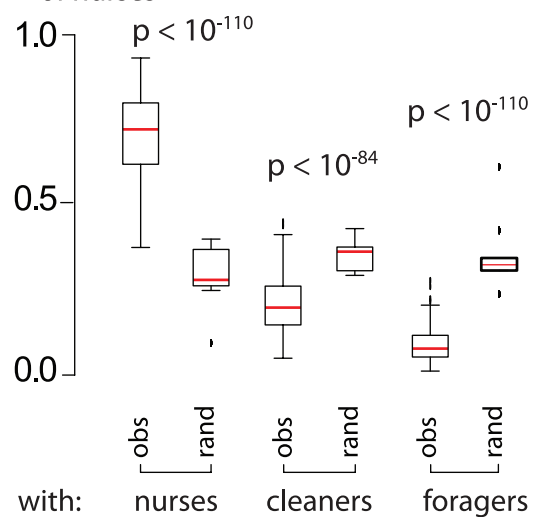

Interaction frequency of cleaners

1.0

Day 9

Interaction frequency of foragers

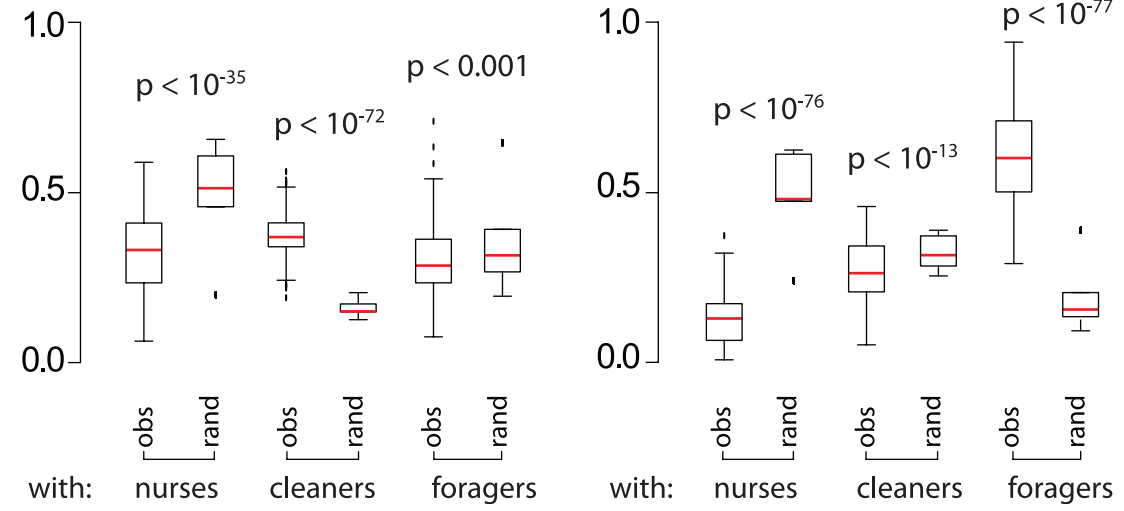

Fig. 1. Colonies are organized in several interconnected social groups within which workers interact frequently. (A) Social networks of colony five on days $1,3,5,7$, and 9 . Edge width is proportional to the number of interactions between pairs of nodes. The darkness of edges is proportional to the average duration of interactions. The network is laid out with the spring

embedded layout from Cytoscape (19). (B) Observed (obs) and theoretically predicted interaction frequencies within- and between-groups of nurses, cleaners, and foragers. Theoretical predictions are based on random (rand) interactions among all ants. $P$ values were from Kruskal-Wallis tests with post-hoc comparisons and Bonferroni corrections for multiple testing. 
A

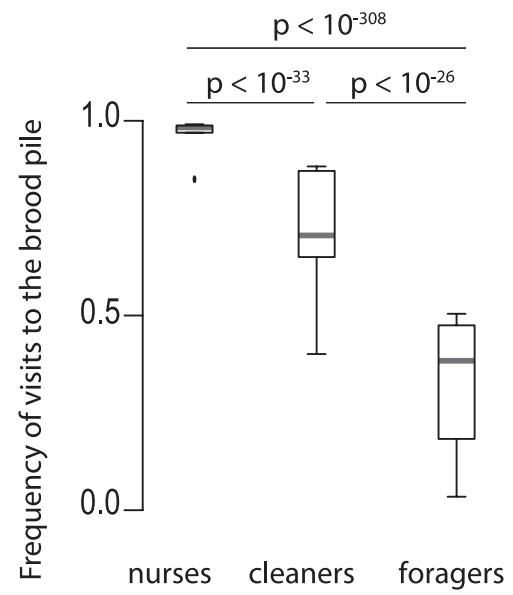

B

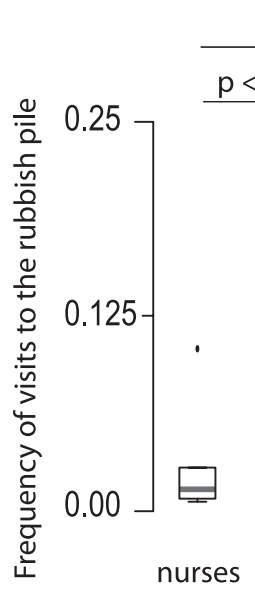

Fig. 2. Behavioral specialization of the three social groups (nurses, cleaners, and foragers). Specialization in (A) nursing, (B) cleaning, and (C) foraging is shown (estimated by the number of visits to the nest entrance,

C

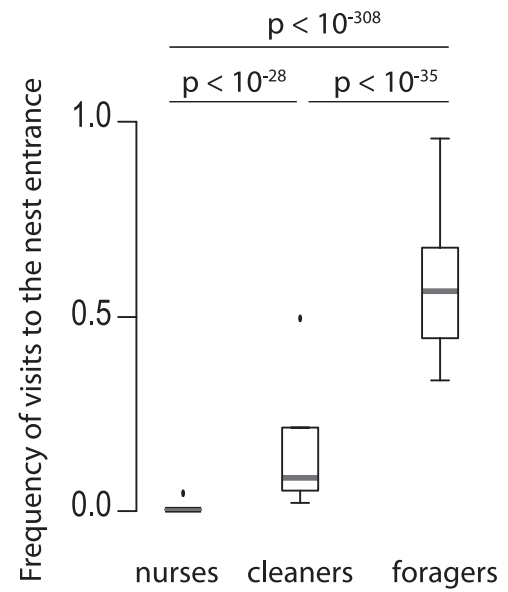

which were strongly correlated with the number of foraging trips). $P$ values represent comparisons between adjacent groups and were from Kruskal-Wallis tests with post-hoc comparisons and Bonferroni corrections for multiple testing. between groups by taking into account the observed between-group spatial segregation (12). Accounting for the spatial distribution led to a greatly improved match between the expected and observed rates of interaction compared with when spatial distribution was not considered (KW: $\chi^{2}=1188.7, P<10^{-259}$ ). Importantly, there were no significant differences between the expected and observed rates of withinand between-group interactions for each of the three groups (KW: $\chi^{2}=1.4, P=0.23$ ) (Fig. 4C), indicating that spatial fidelity and strong spatial assortment of individuals of each of the three groups are the primary mechanisms underlying strong preference for within-group interactions.

The strong link between spatial and social structure further indicates that groups with a more diffuse spatial structure should also have a weaker social group structure. This is exactly what we observed for the cleaners, who have both a more diffuse spatial structure than the two other groups and a lower within-group interaction rate. Similar spatial-social correlations have been observed in reef sharks and bottlenose dolphins, which both form communities that are spatially segregated. However, in contrast to ants, sharks and dolphins actively reinforce their communities by engaging in more interactions with group members than are expected from the spatial structure $(16,17)$.

The presence of spatial and social structure can limit the speed of information flow (18). To quantify the rate of information flow within and between groups, we selected 162 random ants ( 9 per group and colony) as information carriers and calculated a maximum rate of information flow, assuming that all interactions lead to information transfer (12). The analysis shows that as many as $89 \pm 14 \%$ of the ants in the colony can receive the information within 1 hour, independently of who is the information carrier, thereby indicating that information might circulate rapidly. The role of social structure was reflected by a faster spread within than between groups when the information was emitted by nurses

A

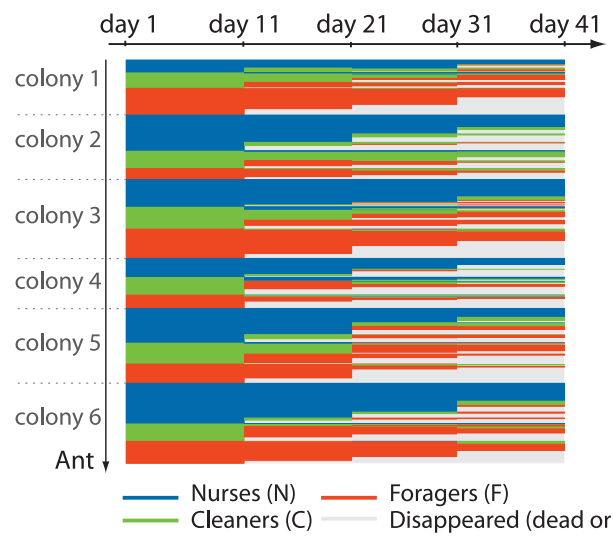

B
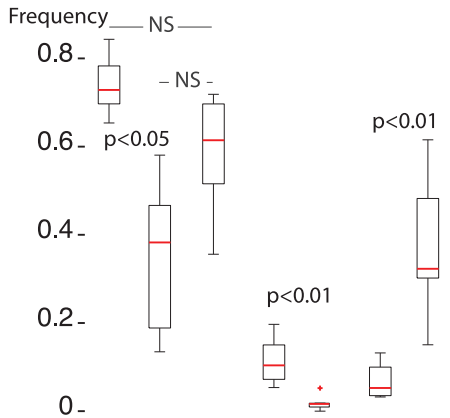

$p<0.001$

0 -

$\mathrm{N}->\mathrm{N} \quad \mathrm{C} \rightarrow \mathrm{C} \quad \mathrm{F}->\mathrm{F} \quad \mathrm{N}->\mathrm{C} \quad \mathrm{N}->\mathrm{F} \quad \mathrm{C}->\mathrm{N} \quad \mathrm{C} \rightarrow \mathrm{F} \quad \mathrm{F}->\mathrm{N} \quad \mathrm{F}->\mathrm{C}$ No group change Group change

Fig. 3. Behavioral trajectories of workers over the $\mathbf{4 1}$ days of the experiments. Workers tend to move from nursing to nest cleaning to foraging. (A) Social group affiliations of each worker for each 11- or 10-day interval. Blue, affiliation to the nurse group; green, cleaning group; orange, foraging group. Workers that disappeared because they died or lost their tag are indicated in white. (B) Frequencies of group changes for workers of each group (N, nurses; $C$, cleaners; $F$, foragers). $P$ values were from Kruskal-Wallis tests with posthoc comparisons and Bonferroni corrections for multiple testing. NS indicates nonsignificant differences.

and foragers (nurses: Wald test $=32.7, P<10^{-7}$; foragers: Wald test $=18.7, P<10^{-4}$ ) (fig. S18, A and $\mathrm{C})$. However, this was not the case when cleaners were the information carriers (Wald test $=0.84$, $P=0.36$ ) (fig. S18B), most likely because they are spatially and topologically in the middle between nurses and foragers (by contrast, nurses and foragers have only cleaners as their neighbors). Thus, although social and spatial structure limit information flow, the extent to which the limits are experienced by the colony depends on who carries the information. These results suggest that spatial structure might function as a regulating mechanism for information flow, division of labor, and colony homeostasis.

Our tracking system allowed us to precisely assess a network of interaction within colonies and uncover the existence of three groups of workers that preferentially interact with individuals of their group and exhibit different behavioral repertoires. The higher-than-expected interaction rate within groups was due to strong differences in the spatial location among individuals of the three groups, highlighting spatial fidelity as a key regulator of network interactions and the spread of information within ant colonies.

References and Notes

1. B. Hölldobler, E. O. Wilson, The Ants (Springer, Berlin, 1990).

2. G. E. Robinson, Annu. Rev. Entomol. 37, 637 (1992).

3. A. F. G. Bourke, N. R. Franks, Social Evolution in Ants (Princeton Univ. Press, Princeton, N], 1995).

4. S. Camazine et al., Self-Organization in Biological Systems (Princeton Univ. Press, Princeton, N], 2003).

5. E. J. H. Robinson, O. Feinerman, N. R. Franks, Proc. Biol. Sci. 276, 4373 (2009)

6. J. M. Jandt, A. Dornhaus, Anim. Behav. 77, 641 (2009). 

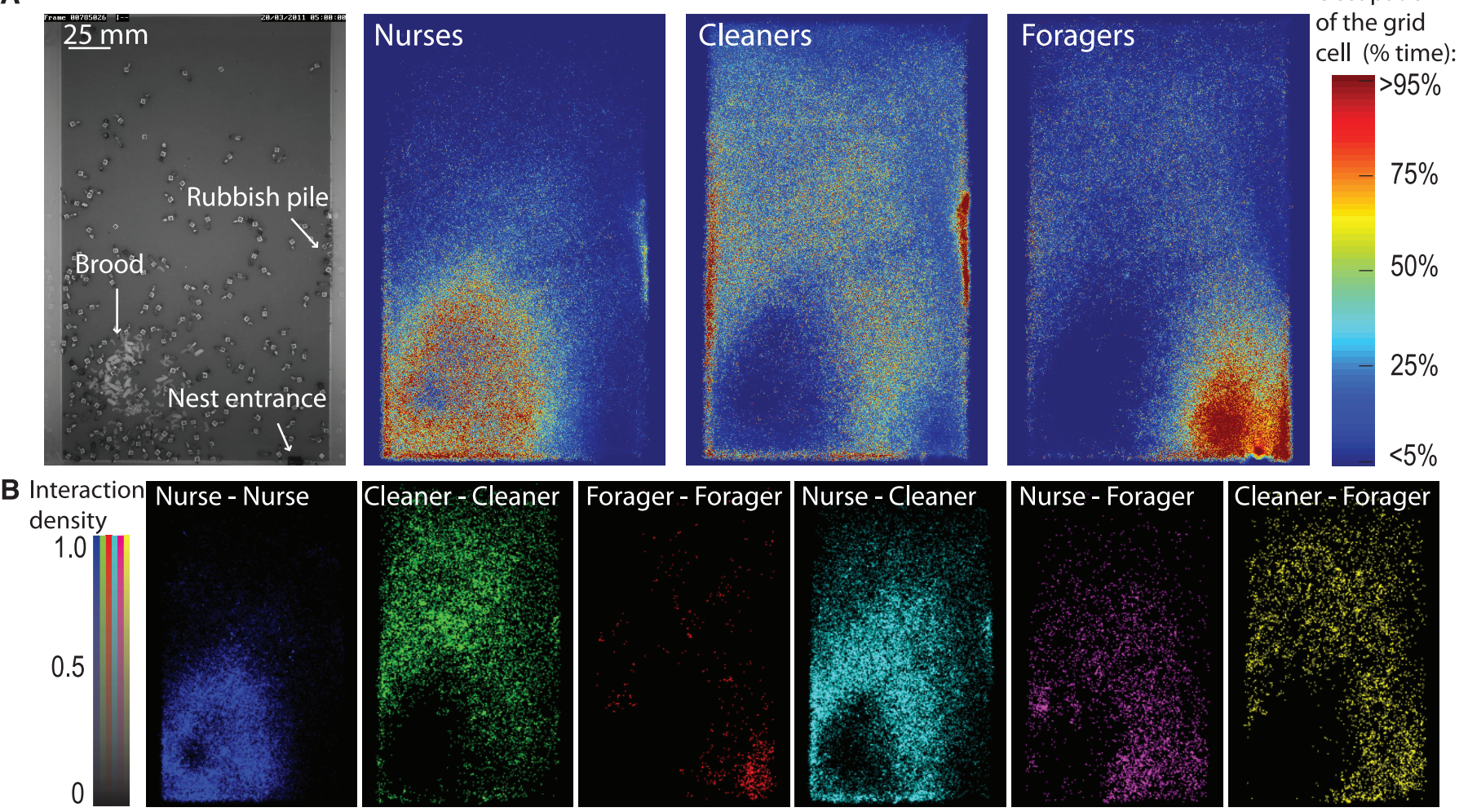

\section{Divergence of interaction} frequency of nurses

\section{Divergence of interaction frequency of cleaners}

\section{Divergence of interaction \\ frequency of foragers}

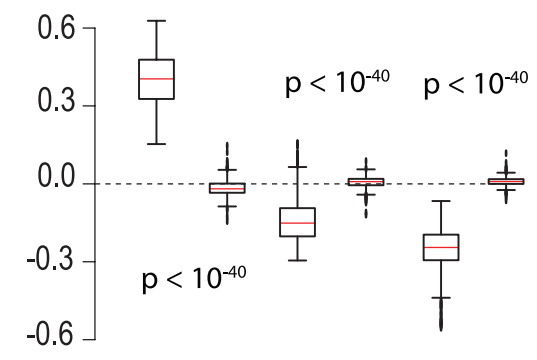

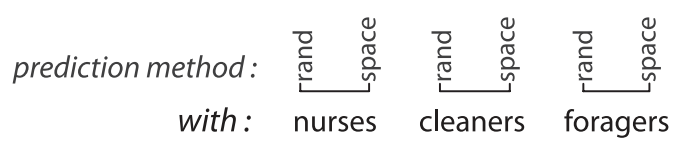
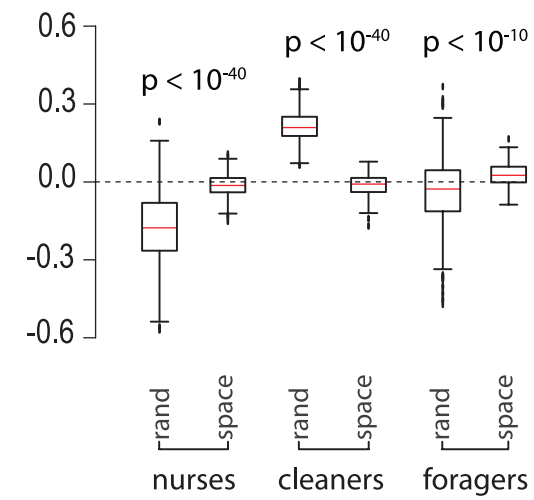

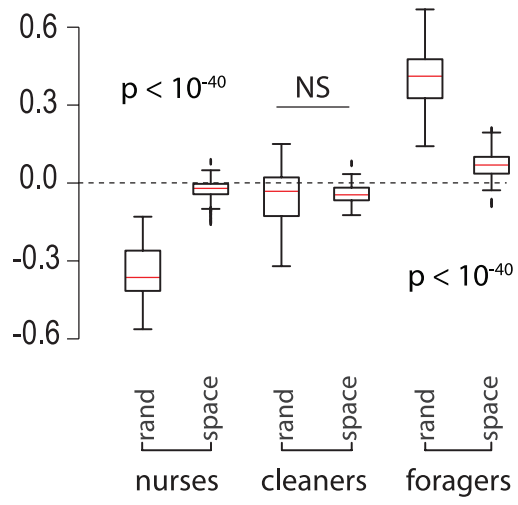

Fig. 4. Spatial segregation of social groups is best explained by the local neighborhood of the ant. (A) Spatial organization of a nest and spatial distribution of nurses, cleaners, and foragers. (B) Spatial distribution of within- and between-group interactions. (C) Divergence between the observed and predicted interaction frequencies. Predictions are generated by assuming random interactions and no spatial segregation among workers from the three groups (i.e., random method) or by taking into account the spatial segregation of workers (i.e., space method). A perfect correspondence between the observed and predicted interaction frequencies is indicated by zero divergence (dotted lines). $P$ values were from Kruskal-Wallis tests.
7. C. R. Smith, A. L. Toth, A. V. Suarez, G. E. Robinson, Nat. Rev. Genet. 9, 735 (2008).

8. B. R. Johnson, Behav. Ecol. Sociobiol. 62, 777 (2008).

9. T. D. Seeley, Behav. Ecol. Sociobiol. 11, 287 (1982).

10. M. Vonshak, A. Shlagman, Isr. J. Entomol. 39, 165 (2009).

11. M. Fiala, "Comparing ARTag and ARToolkit Plus fiducial marker systems," HAVE 2005, IEEE International Workshop on Haptic Audio Visual Environments and Their Applications, Ottawa, 1 to 2 October 2005.

12. Materials and methods are available as supplementary materials on Science Online.

13. M. Rosvall, C. T. Bergstrom, Proc. Natl. Acad. Sci. U.S.A. 105, 1118 (2008).

14. M. A. Seid, J. F. A. Traniello, Behav. Ecol. Sociobiol. 60, 631 (2006).

15. A. Lenoir, Bull. Biol. Fr. Belg. 2-3, 80 (1979).
16. J. Mourier, ]. Vercelloni, S. Planes, Anim. Behav. 83, 389 (2012).

17. D. Lusseau et al., J. Anim. Ecol. 75, 14 (2006).

18. B. Blonder, A. Dornhaus, PLoS ONE 6, e20298 (2011).

19. P. Shannon et al., Genome Res. 13, 2498 (2003).

Acknowledgments: This work was funded by an advanced European Research Council grant, several grants from the Swiss National Science Foundation, the Société Académique Vaudoise, and the Fondation Erna Hamburger. We declare no conflicts of interest. We thank M. Fiala for advice on the use of the ARTag library; A. Hefetz for collecting queens; $B$. Hollis for help with the information spread analysis; R. James for discussions on network analysis; the Swiss Institute of Bioinformatics for data storage; and M. Chapuisat, B. Hollis, T. Kawecki, E. Lucas, N. Stroeymeyt, T. Richardson, and three anonymous reviewers for comments on the manuscript. Electronic schematics and software are available upon request. Data (social networks, behavior, spatial structure, observed and expected interaction frequencies, and information spread) that pertain to the experiments and analyses reported in this paper can be accessed at DOI: 10.5061/dryad.8d8h7.

\section{Supplementary Materials}

www.sciencemag.org/cgi/content/full/science.1234316/DC1 Materials and Methods

Supplementary Text

Figs. S1 to S18

References (20-22)

Movies $\mathrm{S} 1$ to $\mathrm{S} 3$

20 December 2012; accepted 28 March 2013

Published online 18 April 2013;

10.1126/science. 1234316 